\title{
Association of Cardiovascular Risk Assessment with Early Colorectal Neoplasia Detection in Asymptomatic Population: A Systematic Review and Meta-Analysis
}

This article was published in the following Dove Press journal: Clinical Epidemiology

\author{
Yanwei Chen' \\ Xuechen Chen $\mathbb{D}^{2}$ \\ Xi Wang $\mathbb{D}^{2}$ \\ Zhunzhun Liu $\mathbb{D}^{2}$ \\ Haibo Zhou ${ }^{3}$ \\ Shu $\mathrm{Xu}^{4}$ \\ 'Infection Control Department of \\ Shenzhen Hospital of University of \\ Chinese Academy of Sciences, Shenzhen, \\ People's Republic of China; ${ }^{2}$ Medical \\ Faculty Heidelberg, University of \\ Heidelberg, Heidelberg, Germany; \\ ${ }^{3}$ Institute of Pharmaceutical Analysis, \\ College of Pharmacy, Jinan University, \\ Guangzhou, People's Republic of China; \\ ${ }^{4}$ Oncology Department of Shenzhen \\ Hospital of University of Chinese \\ Academy of Sciences, Shenzhen, People's \\ Republic of China
}

\begin{abstract}
Previous studies have shown a strong coexistence of colorectal neoplasia (CRN) and cardiovascular diseases (CVD). This study was aimed to summarize the available evidence on association of CVD risk with early CRN detection in asymptomatic populations. PubMed, Web of Science, and Embase were systematically searched for eligible studies published until Dec 20, 2019. Studies exploring the associations of recommended CVD risk assessment methods (e.g., risk scores, carotid artery plaque, and coronary artery calcium score [CACS]) with risk of CRN were included. Meta-analyses were conducted to determine the overall association of CVD risk with the CRN. A total of 12 studies were finally included. The association of carotid artery plaque with the risk of colorectal adenoma (AD) was weakest (pooled odds ratio [OR)] 1.27, 95\% confidence interval [CI), 1.12, 1.45]. Participants with CACS $>100$ had about 2-fold increased risk of AD than those with $\mathrm{CACS}=0$. The pooled ORs were 3.36 (95\% CI, 2.15, 5.27) and 2.30 (95\% CI, 1.69, 3.13) for the risk of advanced colorectal neoplasia ( $\mathrm{AN}$ ) and $\mathrm{AD}$, respectively, in participants with Framingham risk score (FRS) $>20 \%$, when compared to participants at low risk (FRS $<10 \%$ ). FRS might help identify subgroups at increased risk for AN, but further studies are needed.
\end{abstract}

Keywords: cardiovascular disease, risk assessment, colorectal neoplasia

\section{Introduction}

Both colorectal cancer (CRC) and cardiovascular diseases (CVD) are the leading causes of mortality and morbidity worldwide. ${ }^{1,2}$ Previous studies have shown a strong coexistence of colorectal neoplasia (CRN) and CVD, probably due to the shared risk factors (e.g., smoking, obesity, and metabolic syndrome) and pathophysiological mechanisms (e.g., chronic inflammation and oxidative stress). ${ }^{3-7}$

Current guidelines ${ }^{8-10}$ recommend assessing the CVD risk in healthy people using risk estimation scores such as Framingham Risk Score (FRS), ${ }^{11,12}$ PROCAM, ${ }^{13}$ and the pooled cohort equation, ${ }^{14}$ which are based on individuals' medical history and easily available laboratory data. In addition, assessment of subclinical atherosclerosis by imaging modalities could be added as risk modifiers to help make clinical decisions for borderline- or intermediate-risk adults. ${ }^{8-10}$ Routine use of imaging modalities is not recommended for CVD risk assessment in clinical practice due to the medical costs or invasiveness, but incorporation of imaging data such as the ankle-brachial index (ABI), coronary artery calcium score (CACS), and carotid artery plaques (CAP), could improve the prediction of CVD risk. ${ }^{15-17}$
Correspondence : Shu Xu

Email selflearner@126.com

Clinical Epidemiology 2020:12 865-873 
Various risk scores have also been developed for predicting advanced colorectal neoplasia (AN). ${ }^{18-24}$ Although several studies ${ }^{25,26}$ have reported that elevated blood lipids, the well documented CVD risk factor, and history of CVD were associated with increased risk of CRC, the majority of risk scores developed for AN did not include them into the models. ${ }^{27}$ Recent studies have reported the associations between CVD risk assessment and risk of 1 CRN. Higher FRS, estimating the 10-year risk of developing coronary heart disease (CHD) ${ }^{11,12}$ was significantly associated with the higher risk of AN (FRS $>20 \%$ vs. FRS $<10 \%$, odds ratio [OR], 4.12, 95\% confidence interval [CI]: $1.36-12.52) .{ }^{28} \mathrm{ABI}$ was associated with 13-fold increased risk of $\mathrm{AN}$ in a recent study. ${ }^{29} \mathrm{CAP}$ and CACS were also found to be positively related to the increased risk of adenoma $(\mathrm{AD})$ and $\mathrm{AN}$ in several studies ${ }^{30-33}$.

Given a number of shared risk factors and mechanisms between CVD and CRC, and the emerging epidemiological evidence of association between CVD risk and CRC, there is a possibility that CVD risk assessment could help trigger CRC screening. Therefore, the aim of this review was to provide an overview of the CVD risk assessment methods and their associations with the risk of CRN. Fully understanding of the current knowledge and existing gap might promote better prevention and treatment for CVD and CRC. Circulating and urinary biomarkers have either no or only limited value when added to CVD risk estimation score systems, ${ }^{8,34}$ thus only score models and imaging methods recommended as risk modifiers (ABI, CACS and CAP) in the guidelines ${ }^{8-10}$ were included in this review.

\section{Materials and Methods}

This systematic review was conducted following the procedure recommended by the Cochrane Collaboration ${ }^{35}$ and was reported according to the Preferred Reporting Items for Systematic Reviews and Meta-Analyses (PRISMA) checklist. ${ }^{36}$ Ethical approval and patient informed consent were not necessary, since all the data included in the current study were obtained from previously published studies.

\section{Literature Search Strategies}

PubMed, Embase and Web of Science were searched up to December 20, 2019 to identify the relevant papers. The searched items were presented in the Appendix, which mainly covers expressions for CVD risk score models, recommended imaging modalities, $\mathrm{CRN}$, and discriminatory accuracy or strength of association. After removal of duplicates, titles and abstracts of records were screened according to the inclusion and exclusion criteria. Full texts of the remaining publications and reference lists were scrutinized. Studies that fulfilled the pre-defined criteria were included.

\section{Inclusion and Exclusion Criteria}

We required that included studies meet the following criteria: 1) published as an original research article in a peerreviewed journal; 2) cardiovascular risk has been assessed using either score models or imaging methods recommended as risk modifiers (ABI, CACS and CAP) in the guidelines;(3) only included participants who were considered asymptomatic; 4) reported the association of CVD risk assessment results with the risk of CRN. Studies were excluded if they were published as conference proceedings, dissertations or abstracts only or were not published in English. PICO eligibility criteria for this review were presented in the supplementary Table S1.

\section{Data Extraction}

Two authors (Y.C. and X.C.) independently performed data extraction of all included studies. The following information was abstracted: author, publication year, study period, number of participants, age, number of males, outcome (AD, AN, and so on), data source (medical records, questionnaires or both), CVD risk assessment and association index/discriminatory accuracy (OR, hazard ratio [HR], specificity, sensitivity, or area under the receiver operator characteristic curve values]). In case of any disagreement, consensus was obtained by discussion.

\section{Quality Assessment in Eligible Studies}

Risk of bias and applicability were assessed according to Quality Assessment of Diagnostic Accuracy Studies-2 (QUADAS-2). ${ }^{37}$ QUADAS-2 evaluates the risk level of bias composed of four basic components: 1) patient selection; 2) index test; 3) reference standard; 4) flow and timing. Clinical applicability is also assessed for the first three components. The risk of bias and concerns regarding applicability for each study was then rated as "high," "low," or "unclear."

\section{Statistical Analysis}

We pooled ORs for the same CVD risk assessment index using R statistical software (version 3.6.2) and the R "meta" package (version 4.9-8). For FRS and CACS, ORs were pooled separately for different levels of scores using the lowest level as reference. Two kinds of outcomes (AD and AN) were reported in the studies using FRS for CVD risk assessment, and thus, ORs were pooled separately for different outcomes. Heterogeneity across studies was evaluated 
using Cochrane's Q statistic with $\mathrm{P}$ value and the $\mathrm{I}^{2}$ statistic. If significant heterogeneity was observed $\left(\mathrm{I}^{2}>50 \%\right.$ or $P_{\text {Q-Statistics }}<0.10$ ), a random-effects model was used to calculate pooled estimates, otherwise a fixed-effects model was used. ${ }^{35}$ Two-sided $\mathrm{P}$ values of 0.05 or lower were considered to be statistically significant.

\section{Results}

\section{Literature Search Results}

A total of 7391 records were obtained in the initial search, including 1124 citations from PubMed, 4283 citations from Embase, and 1984 citations from Web of Science. After removal of duplicates $(n=1609)$ and exclusion due to our pre-defined criteria $(n=5727), 55$ records were qualified for full-text assessment. Forty-four records were excluded due to the inclusion and exclusion criteria. Finally, a total of 12 studies $^{28-33,38-43}$ including one study which was identified through cross-references were included. The detailed information of the selection process was presented in Figure 1.

\section{Study Characteristics}

Table 1 summarized the basic characteristics of the included studies published between 2011 and 2019. Of the 12 included studies, nine were from Korea, and the other three studies were from Japan, Austria, and Turkey, respectively. The study periods stretched from 2006 to 2017, with sample sizes ranging from 192 to 4871. Only one was designed as a prospective study, ${ }^{41}$ and the others were cross-sectional studies. Most studies included participants aged older than 50 years, and only one study enrolled subjects aged $<50$ years. ${ }^{32}$ In addition, most studies were predominantly in men with proportions of males among participants ranging from $51.2 \%$ to $91.1 \%$. Four CVD risk assessment methods (ABI, CAP, CACS and FRS) were used in the included studies. All studies explored the role of CVD risk assessment method on the detection of $\mathrm{AD}$, and some of them also focused on colorectal high risk adenoma $^{30,32}$ and $\mathrm{AN} .^{28,29,38,42,43}$
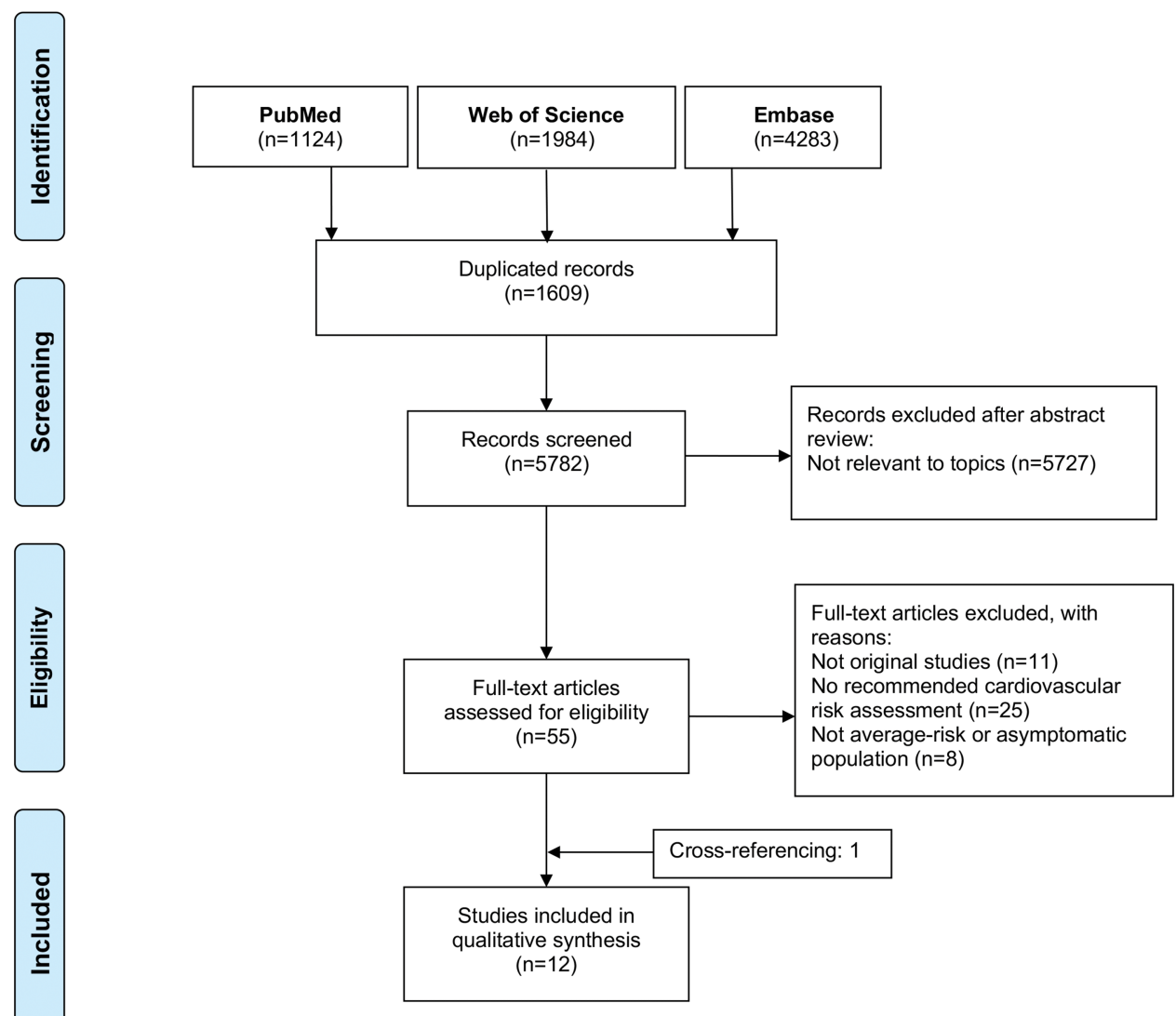

Figure I Flowchart of inclusions of studies about relation of CVD risk to CRN.

Note: Adapted from Moher D, Liberati A, Tetzlaff J, et al.Preferred reporting items for systematic reviews and meta-analyses: the PRISMA statement. PLoS Med. 2009;6:7. Creative Commons license and disclaimer available from: http://creativecommons.org/licenses/by/4.0/legalcode. ${ }^{36}$

Abbreviations: CVD, cardiovascular disease; CRN, colorectal neoplasia. 
Table I Basic Characteristics of Included Studies About Relation of CVD Risk to Colorectal Neoplasia

\begin{tabular}{|c|c|c|c|c|c|c|c|c|c|}
\hline Study & Country & $\begin{array}{l}\text { Study } \\
\text { Period }\end{array}$ & $\begin{array}{l}\text { Number of } \\
\text { Participants }\end{array}$ & $\begin{array}{l}\text { Age } \\
\text { (Years, } \\
\text { Mean } \pm S D)\end{array}$ & $\begin{array}{l}\text { Male, } \\
\text { N (\%) }\end{array}$ & Outcome $^{c}$ & $\begin{array}{l}\text { Data } \\
\text { Source }\end{array}$ & $\begin{array}{l}\text { Cecum } \\
\text { Intubation } \\
\text { Rate }^{\text {d }}\end{array}$ & $\begin{array}{l}\text { CVD Risk } \\
\text { Assessment }\end{array}$ \\
\hline Yamaji Y, $2014^{29}$ & Japan & $2006-2010$ & 907 & $57.3 \pm 8.7$ & 652 (71.9) & $A D, A N$ & $M R+Q$ & $100 \%$ & $\mathrm{ABI}$ \\
\hline Kim J, $2019^{30}$ & Korea & $2012-2016$ & 4871 & $54.7 \pm 8.4$ & $2492(51.2)$ & $A D, H R A$ & $M R+Q$ & NR & CAP \\
\hline $\mathrm{Kim} \mathrm{H}, 2018^{31}$ & Korea & $2013-2017$ & 548 & $53.0^{\mathrm{b}}$ & $412(75.2)$ & $A D$ & $M R+Q$ & $100 \%$ & CAP \\
\hline Cha JM, $2011^{38}$ & Korea & 2006-2009 & 192 & 56 (Median) & $115(59.9)$ & $A D, A N$ & $M R$ & $N R$ & CAP \\
\hline Yun KE, $2018^{32}$ & Korea & $2010-2011$ & 4859 & $41.0 \pm 7.0$ & $4354(89.6)$ & $A D, H R A$ & $M R+Q$ & $100 \%$ & CACS \\
\hline Choi SH, $2015^{33}$ & Korea & $2009-2014$ & 398 & $56.8 \pm 8.1$ & $290(72.9)$ & $A D$ & $M R+Q$ & NR & CACS \\
\hline Yang $\mathrm{MH}, 2013^{39}$ & Korea & 2006-2009 & 3092 & $52.5 \pm 7.1$ & 2807 (9l.I) & $A D$ & $M R$ & $100 \%$ & CACS \\
\hline $\mathrm{Kim}, \mathrm{HB}, 2014^{40}$ & Korea & $2007-2010$ & 1637 & $51.7 \pm 9.5$ & 981 (59.9) & $A D$ & MR & NR & CACS \\
\hline Lee YJ, $2019^{\mathrm{a} 4 \mathrm{l}}$ & Korea & 2006-2017 & 754 & $52.6^{\mathrm{b}}$ & 544 (72.1) & $A D$ & $M R+Q$ & $\geq 95 \%$ & CACS \\
\hline Lee JY, $2013^{42}$ & Korea & 2010 & 3144 & $50.5 \pm 7.8$ & $2068(65.8)$ & $A D, A N$ & $M R+Q$ & $100 \%$ & FRS \\
\hline Niederseer D, $2018^{43}$ & Austria & $2010-2014$ & 1990 & $58.7 \pm 9.7$ & $982(49.4)$ & $A D, A N$ & $M R+Q$ & NR & FRS \\
\hline Basyigit S, $2015^{28}$ & Turkey & 2014 & 235 & $60.3 \pm 12.6$ & $126(53.0)$ & $A D, A N$ & $M R+Q$ & $100 \%$ & FRS \\
\hline
\end{tabular}

Notes: ${ }^{\text {alt }}$ is a retrospective follow-up study, and all the other studies are cross-sectional; ${ }^{\text {bSD }}$ was not reported; ${ }^{\mathrm{C} D e t e c t e d ~ b y ~ c o l o n o s c o p i e s ~ i n ~ a l l ~ i n c l u d e d ~ s t u d i e s ; ~}{ }^{\mathrm{d}} \mathrm{I00 \%}$ cecum intubation rate: participants with failure of cecum intubation were excluded; NR, not reported; studies mentioned that colonoscopies were extended to cecum in the methods section, but did not reported the success rate of cecum intubation.

Abbreviations: ABI, ankle-brachial index; AD; colorectal adenoma; AN, advanced colorectal neoplasia; CACS, coronary artery calcium score; CAP, carotid artery plaque; CVD, cardiovascular disease; FRS, Framingham risk score; HRA, high risk adenoma; MR, medical records; NR, not reported; Q, questionnaires; SD, standard deviation.

\section{Quality Assessment of Studies}

The results for the quality of included studies using the QUADAS-2 tool are presented in Table 2. Regarding patient selection, one study by Kim et al did not provide detailed information about patient selection. ${ }^{31}$ Thus, the risk of bias and applicability concerns were rated unclear for this domain in this study. Otherwise, no major risk of bias or applicability concerns were identified.

\section{Association of CVD Risk Assessed by Different Methods with CRC Risk}

Table 3 described the details of the CVD risk assessment methods in the included studies. ABI was associated with 13 -fold $(95 \% \mathrm{CI}, 2.69,62.7)$ increased risk of $\mathrm{AN}{ }^{29}$ Three studies reported the weak association between $\mathrm{CAP}$ and risk of $\mathrm{AD} .^{30,31,38}$ One of them also showed an increased risk of AN in the participants with CAP, but the results were not statistically significant (OR, 3.10,

Table 2 Risk of Bias and Applicability Judgements in QUADAS-2

\begin{tabular}{|c|c|c|c|c|c|c|c|c|}
\hline \multirow[t]{2}{*}{ Study } & \multicolumn{4}{|c|}{ Risk of Bias } & \multicolumn{3}{|c|}{ Applicability Concerns } & \multirow[t]{2}{*}{ Total } \\
\hline & $\begin{array}{l}\text { Patient } \\
\text { Selection }\end{array}$ & $\begin{array}{l}\text { Index } \\
\text { Test }\end{array}$ & $\begin{array}{l}\text { Reference } \\
\text { Standard }\end{array}$ & $\begin{array}{l}\text { Flow and } \\
\text { Timing }\end{array}$ & $\begin{array}{l}\text { Patient } \\
\text { Selection }\end{array}$ & $\begin{array}{l}\text { Index } \\
\text { Test }\end{array}$ & $\begin{array}{l}\text { Reference } \\
\text { Standard }\end{array}$ & \\
\hline Yamaji Y, 2014 $4^{29}$ & $\sqrt{ }$ & $\sqrt{ }$ & $\sqrt{ }$ & $\sqrt{ }$ & $\sqrt{ }$ & $\sqrt{ }$ & $\sqrt{ }$ & 7 \\
\hline Kim J, 201930 & $\sqrt{ }$ & $\sqrt{ }$ & $\sqrt{ }$ & $\sqrt{ }$ & $\sqrt{ }$ & $\sqrt{ }$ & $\sqrt{ }$ & 7 \\
\hline $\mathrm{Kim} \mathrm{H}, 2018^{31}$ & $?$ & $\sqrt{ }$ & $\sqrt{ }$ & $\sqrt{ }$ & $?$ & $\sqrt{ }$ & $\sqrt{ }$ & 5 \\
\hline Cha JM, 20II $I^{38}$ & $\sqrt{ }$ & $\sqrt{ }$ & $\sqrt{ }$ & $\sqrt{ }$ & $\sqrt{ }$ & $\sqrt{ }$ & $\sqrt{ }$ & 7 \\
\hline Yun KE, $2018^{32}$ & $\sqrt{ }$ & $\sqrt{ }$ & $\sqrt{ }$ & $\sqrt{ }$ & $\sqrt{ }$ & $\sqrt{ }$ & $\sqrt{ }$ & 7 \\
\hline Choi SH, $2015^{33}$ & $\sqrt{ }$ & $\sqrt{ }$ & $\sqrt{ }$ & $\sqrt{ }$ & $\sqrt{ }$ & $\sqrt{ }$ & $\sqrt{ }$ & 7 \\
\hline Yang $\mathrm{MH}, 2013^{39}$ & $\sqrt{ }$ & $\sqrt{ }$ & $\sqrt{ }$ & $\sqrt{ }$ & $\sqrt{ }$ & $\sqrt{ }$ & $\sqrt{ }$ & 7 \\
\hline $\mathrm{Kim}, \mathrm{HB}, 2014^{40}$ & $\sqrt{ }$ & $\sqrt{ }$ & $\sqrt{ }$ & $\sqrt{ }$ & $\sqrt{ }$ & $\sqrt{ }$ & $\sqrt{ }$ & 7 \\
\hline Lee YJ, 2019 & $\sqrt{ }$ & $\sqrt{ }$ & $\sqrt{ }$ & $\sqrt{ }$ & $\sqrt{ }$ & $\sqrt{ }$ & $\sqrt{ }$ & 7 \\
\hline Lee JY, $2013^{42}$ & $\sqrt{ }$ & $\sqrt{ }$ & $\sqrt{ }$ & $\sqrt{ }$ & $\sqrt{ }$ & $\sqrt{ }$ & $\sqrt{ }$ & 7 \\
\hline Niederseer D, $2018^{43}$ & $\sqrt{ }$ & $\sqrt{ }$ & $\sqrt{ }$ & $\sqrt{ }$ & $\sqrt{ }$ & $\sqrt{ }$ & $\sqrt{ }$ & 7 \\
\hline Basyigit S, $2015^{28}$ & $\sqrt{ }$ & $\sqrt{ }$ & $\sqrt{ }$ & $\sqrt{ }$ & $\sqrt{ }$ & $\sqrt{ }$ & $\sqrt{ }$ & 7 \\
\hline Total & 11 & 12 & 12 & 12 & 11 & 12 & 12 & \\
\hline
\end{tabular}

Notes: “_”, High risk; “ل”, Low Risk; “?”, Unclear Risk. 
Table 3 Details of the CVD Risk Assessment Methods in the Included Studies About Relation of CVD Risk to Colorectal Neoplasia

\begin{tabular}{|c|c|c|c|c|}
\hline Study & Categories $^{b}$ & Outcome & OR & {$[95 \% \mathrm{Cl}]$} \\
\hline \multirow[t]{2}{*}{ Yamaji Y, $2014^{29}$} & Abnormal $A B I$ & $A D$ & 1.76 & {$[0.55,5.63]$} \\
\hline & Abnormal ABI & AN & 13.0 & {$[2.69,62.7]$} \\
\hline \multirow[t]{2}{*}{ Kim J, $2019^{30}$} & CAP: Yes & $A D$ & 1.24 & {$[1.08,1.43]$} \\
\hline & CAP: Yes & HRA & 1.35 & {$[1.10,1.65]$} \\
\hline $\mathrm{Kim} \mathrm{H}, 2018^{31}$ & CAP: Yes & $A D$ & 1.70 & {$[1.04,2.76]$} \\
\hline \multirow[t]{2}{*}{ Cha JM, $2011^{38}$} & CAP: Yes & $A D$ & 1.35 & {$[0.68,2.68]$} \\
\hline & CAP: Yes & AN & 3.10 & {$[0.83,11.67]$} \\
\hline \multirow[t]{6}{*}{ Yun KE, $2018^{32}$} & CACS: $>0$ & $A D$ & 1.37 & {$[1.09,1.70]$} \\
\hline & CACS: $1-100$ & $A D$ & 1.26 & {$[1.00,1.60]$} \\
\hline & CACS: >100 & $A D$ & 2.07 & {$[1.31,3.26]$} \\
\hline & CACS: $>0$ & HRA & 1.98 & {$[1.23,3.19]$} \\
\hline & CACS: $1-100$ & HRA & 1.90 & {$[1.15,3.13]$} \\
\hline & CACS: $>100$ & HRA & 2.54 & {$[1.10,5.89]$} \\
\hline \multirow[t]{3}{*}{ Choi SH, $2015^{33}$} & CACS: $>0$ & $A D$ & 1.66 & {$[1.05,2.64]$} \\
\hline & CACS: $1-100$ & $A D$ & 1.80 & {$[1.06,3.03]$} \\
\hline & CACS: $>100$ & $A D$ & 1.95 & {$[1.05,3.63]$} \\
\hline Yang $\mathrm{MH}, 2013^{39}$ & CACS: $>0$ & $A D$ & 1.35 & {$[1.12,1.61]$} \\
\hline \multirow[t]{3}{*}{$\mathrm{Kim}, \mathrm{HB}, 2014^{40}$} & CACS: $1-17$ & $A D$ & 1.44 & {$[0.91,2.33]$} \\
\hline & CACS: $18-105$ & $A D$ & 1.88 & {$[1.15,3.01]$} \\
\hline & CACS: $\geq 106$ & $A D$ & 3.61 & {$[2.23,5.74]$} \\
\hline Lee YJ, 2019 $9^{\mathrm{a} 41}$ & CACS: $>0$ & $A D$ & HR: 1.29 & {$[0.80,2.08]$} \\
\hline \multirow[t]{5}{*}{ Lee JY, $2013^{42}$} & $\begin{array}{l}\text { FRS: } \\
\text { Intermediate }\end{array}$ & & & {$[1.34,2.05]$} \\
\hline & FRS: High & $A D$ & 2.26 & {$[1.53,3.35]$} \\
\hline & FRS: & AN & 1.26 & {$[0.85,1.87]$} \\
\hline & Intermediate & & & \\
\hline & FRS: High & AN & 3.31 & {$[1.94,5.65]$} \\
\hline \multirow[t]{4}{*}{$\begin{array}{l}\text { Niederseer D, } \\
2018^{43}\end{array}$} & $\begin{array}{l}\text { FRS: } \\
\text { Intermediate }\end{array}$ & $A D$ & 2.34 & \\
\hline & FRS: High & $A D$ & 2.69 & {$[1.47,4.90]$} \\
\hline & FRS: & AN & 2.93 & {$[1.82,4.71]$} \\
\hline & FRS: High & AN & 2.86 & {$[0.84,9.7 \mathrm{I}]$} \\
\hline \multirow[t]{4}{*}{ Basyigit S, $2015^{28}$} & $\begin{array}{l}\text { FRS: } \\
\text { Intermediate }\end{array}$ & $A D$ & 2.89 & {$[1.52,5.49]$} \\
\hline & FRS: High & $A D$ & 1.74 & {$[0.64,4.12]$} \\
\hline & $\begin{array}{l}\text { FRS: } \\
\text { Intermediate }\end{array}$ & AN & 0.04 & {$[0.09,2.01]$} \\
\hline & FRS: High & AN & 4.12 & {$[1.36,12.52]$} \\
\hline
\end{tabular}

Notes: ${ }^{a}$ In participants without adenoma, CACS $>0$ at baseline (compared to CACS $=0$ ) increased the risk of colorectal adenoma at follow-up colonoscopy (HR, I.29, 95\% Cl, 0.80, 2.08). ${ }^{\mathrm{b}}$ The lowest level was defined as reference. Abbreviations: $A B I$, ankle-brachial index; $A D$, colorectal adenoma; $A N$, advanced colorectal neoplasia; CACS, coronary artery calcium score; CAP, carotid artery plaque; FRS, Framingham risk score; HRA, high risk adenoma; HR, hazard ratio; OR, odds ratio; $\mathrm{Cl}$, confidence interval.
$95 \% \mathrm{CI}, 0.83,11.67) .{ }^{38}$ In addition, the presence of CAP was associated with increased risk of colorectal high risk adenoma (OR, 1.35, 95\% CI, 1.10, 1.65). Four studies reported ORs for different levels of CACS with CACS $=0$ as reference. ${ }^{32,33,39,40}$ Highest CACS levels seemed to be associated with the increased risk of $\mathrm{AD}$ with $\mathrm{OR}$ ranging from 1.95 to 3.61. The 10-year CHD risk estimated by FRS was categorized as low risk $(<10 \%)$, intermediate risk $(10 \%--20 \%)$, and high risk $(\geq 20 \%) .{ }^{44}$ Participants with high risk of 10-year CHD had increased risk of either AD or AN. In the study by Basyigit et al, participants at high CHD risk had about 4-fold (OR, 4.12, 95\% CI, 1.36, 12.52) increased risk of $A N{ }^{28}$

\section{Meta-analyses of Available ORs for Different CVD Risk Assessment Methods}

Meta-analyses were performed in the studies that provided ORs and their $95 \%$ CIs for the same CVD risk assessment index. The association of CAP with the risk of $\mathrm{AD}$ was weakest (the pooled OR, 1.27, 95\% CI, 1.12, 1.45). A medium level of CACS (CACS: 1-100) was associated with 1.34-fold increased risk of $\mathrm{AD}$ when compared to the lowest category of CACS (CACS $=0$ ). Participants with CACS $>100$ had an increased risk of $\mathrm{AD}$, and the pooled OR was 2.03 ( $95 \%$ CI, 1.40, 2.93). The pooled ORs were 3.36 (95\% CI, 2.15, 5.27) and 2.30 (95\% CI, 1.69, 3.13) for the risk of $\mathrm{AN}$ and $\mathrm{AD}$, respectively, in participants with high CHD risk (FRS $>20 \%$ ) when compared to participants at low CHD risk $(\mathrm{FRS}<10 \%)$. Further details were presented in Table 4 and in the Supplementary Figures S1-8.

\section{Discussion}

This systematic review summarized the associations of recommended CVD risk assessment methods with risk of CRN in asymptomatic populations. A total of 12 studies including four different methods were identified. Among these methods, FRS was most strongly associated with risk of both AN and AD. Participants with FRS $>20 \%$ have about 3.4-fold and 2.3-fold increased risk of AN and $\mathrm{AD}$, respectively, when compared to participants at low CHD risk $(\mathrm{FRS}<10 \%)$. Only one study ${ }^{29}$ reported that abnormal ABI greatly increased the risk of AN, thus it was not included in the meta-analysis.

Both CRC and CVD are thought to develop via a process of insulin resistance, inflammation and oxidative 
Table 4 Meta-Analysis of Odds Ratios for Different CVD Risk Assessment Tools

\begin{tabular}{|l|l|l|l|l|}
\hline Study & CVD Risk Assessment & Categories $^{\mathbf{a}}$ & Outcome & OR (95\% CI) \\
\hline $30,31,38$ & CAP & Yes vs. No & AD & $1.27(I .12,1.45)$ \\
$32,33,39,40$ & CACS & CACS: $>0$ vs. CACS $=0$ & AD & $1.42(I .24,1.6 I)$ \\
32,33 & CACS & CACS: I-I00 vs. CACS $=0$ & AD & $1.34(1.08,1.66)$ \\
32,33 & CACS & CACS: $>100$ vs. CACS $=0$ & AD & $2.03(1.40,2.93)$ \\
$28,42,43$ & FRS & Intermediate vs. Low risk & AD & $2.09(1.55,2.83)$ \\
$28,42,43$ & FRS & High vs. low risk & AD & $2.30(1.69,3.13)$ \\
$28,42,43$ & FRS & Intermediate vs. Low risk & AN & $0.71(0.19,2.73)$ \\
$28,42,43$ & FRS & High vs. low risk & AN & $3.36(2.15,5.27)$ \\
\hline
\end{tabular}

Note: ${ }^{\text {a }}$ The lowest level was defined as reference.

Abbreviations: AD, colorectal adenoma; AN, advanced colorectal neoplasia; CACS, coronary artery calcium score; CAP, carotid artery plaque; CVD, cardiovascular disease; FRS, Framingham risk score; OR, odds ratio; $\mathrm{Cl}$, confidence interval.

stress, ${ }^{7,45-47}$ which might partially explain why they share a number of risk factors (e.g., alcohol consumption, tobacco use, physical activity, use of anti-inflammatory agents, obesity and diabetes mellitus). ${ }^{45,48}$ In addition, several cellular metabolism-related pathways (eg, AMPK and PPAR- $\gamma$ ), signaling pathways (eg, Wnt signaling pathway) and genetic pathways (eg, LRP6 mutation and TCF7L2 polymorphism), are not only associated with accelerated atherosclerosis and an increased risk of CVD, but also linked to cancer development and progression. ${ }^{7}$ Better understanding of these overlaps might promote shared management of prevention and treatment for both disorders.

In this review, the strength of associations between identified CVD risk assessment methods and the risk of CRN was generally weak except FRS, which was modestly associated with risk of AN (FRS $>20 \%$ vs. FRS $<10 \%$ ). FRS was calculated based on age, total cholesterol, high-density lipoprotein cholesterol, smoking status, systolic blood pressure and treatment of blood pressure, which are typically available in the medical records. $^{44}$ Compared to the more sophisticated risk calculators $^{23,24,49}$ for predicting $\mathrm{AN}$, which need variables such as physical activity, red meat intake and vegetable consumption, FRS has relatively higher generalizability and lower recall bias. A recent study has recommended the combined preventive, screening, and research efforts in the prevention of both CVD and cancer. ${ }^{50}$ If participants with high-risk of CVD predicted by FRS could be recommended to have a screening for $\mathrm{CRN}$, which will help increase compliance and uptake of CRC screening as persons who are aware of their increased risk are more likely to comply with expert recommendations. Furthermore, it also maximizes the medical values of the information participants obtain from a clinical examination or risk assessment, and thus reduces the time and costs for health care.

However, there are some issues that merit our attention. Firstly, the included studies are all cross-sectional, which limits the comparisons between FRS and the previously developed risk prediction models for CRC. Secondly, FRS has its own limitations. FRS only estimates 10 -year CHD risk for all individuals 40 years or older but not the overall CVD risk. In addition, it is developed based on the American population, while most of study participants are Asians in the included studies. Studies have shown that FRS overestimated CVD risk in the Asian cohorts. ${ }^{51-54}$ At last, the included studies tended to yield results with wide CI probably due to the limited number of participants. The wider the CI, the less the precision. In summary, higher CVD risk might trigger concurrent CRC screening, which should be further validated on large-scale studies, and future studies could consider about using the overall CVD risk score models developed from data of local cohorts to predict the risk of CRC.

As for imaging data, the association of CAP or CACS with risk of $\mathrm{AD}$ is not strong enough that imaging index alone might not be useful for informing early detection of CRN. Similarly, routine screening with imaging modalities to predict future cardiovascular events is generally not recommended in clinical practice, but use of these imaging techniques has been shown to improve CVD risk assessment and serve as a guide for initiating preventive therapies. ${ }^{8-10}$ A high CACS can help modify the predicted risk obtained from FRS alone, especially among patients in the intermediate-risk category. ${ }^{16} \mathrm{Up}$ to now, only one risk score developed in the Multi-Ethnic Study of Atherosclerosis (MESA) study used both CACS and 
traditional risk factors to predict the 10 -year CHD risk. ${ }^{55}$ Inclusion of CACS in the MESA risk score offered significant improvements in risk prediction (C-statistic 0.80 vs. $0.75 ; \mathrm{p}<0.0001)$. Factors in the risk models like smoking behaviors and blood lipids are closely related to the incidence and progression of CVD, but they are not direct markers of current status of atherosclerosis. This might help explain why the performance of risk models is improved by adding markers with anatomical delineation through imaging technology. Accounting for the higher performance of the combined use of risk scores and imaging tools on CVD risk assessment, further studies could consider about exploring the association of combined form of them with the risk of CRC.

We also observed that less than half of included studies reported the associations of CVD risk with both risk of AN and $\mathrm{AD} .^{28,29,38,42,43}$ Colonoscopy is considered to as a valid primary screening tool for $\mathrm{CRC}$, and is able to detect both $\mathrm{AD}$ and AN. The lower prevalence of AN and the limited number of participants in several included studies might limit the power to explore the relation of AN with CVD risk, which could partly explain why most of studies did not include AN as outcome. Therefore, the findings should be carefully interpreted and further validated on large-scale studies.

Our study has some strengths. Comprehensive search strategies along with well-defined eligibility criteria were used to help identify relevant articles. In addition, two reviewers independently extracted data and assessed the risk of bias in the included studies. However, several limitations should also be addressed. Firstly, the current metaanalysis was based on observational studies, there were the possibilities of potential effects of unknown or residual confounding factors on our results. Secondly, as we only considered about established CVD risk models and recommended imaging modalities, the potential of other CVD risk assessment index on the detection of CRN was not summarized and compared in this study. However, it is also reasonable to just include these methods, since their feasibility and performance for CVD risk prediction have been well approved in the clinical practice. Thirdly, cut off values and group comparisons for the same CVD risk assessment method varied in the included studies, which limits the synthesis of results. For example, the cut off values for CACS are the tertiles of CACS in the study by Kim et al. ${ }^{40}$ However, CACS was categorized into three groups with cut off values at 0 and 100 in the other studies. ${ }^{32,33}$ Therefore, less studies were included in the meta-analysis, which might influence the accuracy of the pooled results. Lastly, most of studies were conducted in Asian populations, which is an inherent limitation of the included studies. Thus, our findings might not be applicable to other populations, and needs to be externally validated in racially diverse populations.

\section{Conclusions}

To our knowledge, this is the first review that applies meta-analyses to determining the overall association of recommended $\mathrm{CV}$ risk assessment methods with the risk of $\mathrm{CRN}$ in the asymptomatic population. FRS calculated based on shared risk factors of CVD and CRC shows potential to help identify subgroups at increased risk for AN. Whether the combination of FRS and imaging index is useful for the optimal evaluation of CRN risk remains to be solved in the future studies. CVD risk might inform CRC screening, which needs more research in the future to validate its feasibility and effectiveness.

\section{Abbreviations}

ABI, ankle-brachial index; AD, colorectal adenoma; AN, advanced colorectal neoplasia; CACS, coronary artery calcium score; CAP, carotid artery plaque; CHD, coronary heart disease; CI, confidence interval; CRC, colorectal cancer; CRN, colorectal neoplasia; CVD, cardiovascular disease; FRS, Framingham risk score; HR, hazard ratio; HRA, high risk adenoma; MR, medical records; NR, not reported; OR, odds ratio; PRISMA, Preferred Reporting Items for Systematic Reviews and Meta-Analyses; QUADAS-2, Quality Assessment of Diagnostic Accuracy Studies-2; Q, questionnaires; SD, standard deviation.

\section{Funding}

This research was funded by National Natural Science Foundation of China, grant number 81773684.

\section{Disclosure}

The authors report no conflicts of interest in this work.

\section{References}

1. Bray F, Ferlay J, Soerjomataram I, Siegel RL, Torre LA, Jemal A. Global cancer statistics 2018: GLOBOCAN estimates of incidence and mortality worldwide for 36 cancers in 185 countries. CA Cancer J Clin. 2018;68(6):394-424. doi:doi:10.3322/caac.21492

2. Joseph P, Leong D, McKee M, et al. Reducing the global burden of cardiovascular disease, part 1: the epidemiology and risk factors. Circ Res. 2017;121(6):677-694. doi:doi:10.1161/CIRCRESAHA.117.308903

3. Chan AOO, Man HJ, Kwok FL, et al. Prevalence of colorectal neoplasm among patients with newly diagnosed coronary artery disease. J Am Med Assoc. 2007;298(12):1412. doi:doi:10.1001/jama.298.12. 1412 
4. Chan AOO, Lam KF, Tong T, et al. Coexistence between colorectal cancer/adenoma and coronary artery disease: results from 1382 patients. Aliment Pharmacol Ther. 2006;24(3):535-539. doi: doi:10.1111/j.1365-2036.2006.02958.x

5. Wang SC, Schulman-Marcus J, Fantauzzi J, et al. Colon cancer laterality is associated with atherosclerosis and coronary artery disease. J Gastrointest Oncol. 2019. doi:doi:10.21037/jgo.2018.09.18

6. Kahr PC, Hammerl S, Huber-Schönauer U, et al. Atrial fibrillation: a new indicator for advanced colorectal neoplasia in screening colonoscopy. J Clin Med. 2019;8(7):1083. doi:doi:10.3390/jcm8071083

7. Masoudkabir F, Sarrafzadegan N, Krahn A, et al. Cardiovascular disease and cancer: evidence for shared disease pathways and pharmacologic prevention Cardiovascular disease and cancer: evidence for shared disease pathways and pharmacologic prevention HHS Public Access. Atherosclerosis. 2017;263:343-351. doi:doi:10.1016/ j.atherosclerosis.2017.06.001

8. Piepoli MF, Hoes AW, Agewall S, et al. European guidelines on cardiovascular disease prevention in clinical practice: the sixth joint task force of the European society of cardiology and other societies on cardiovascular disease prevention in clinical practice (constituted by representatives of 10 societies and by invited experts) developed with the special contribution of the European Association for Cardiovascular Prevention \& Rehabilitation (EACPR). Atherosclerosis. 2016;2016 (252):207-274. doi:doi:10.1016/j.atherosclerosis.2016.05.037

9. Arnett DK, Blumenthal RS, Albert MA, et al. 2019 ACC/AHA guideline on the primary prevention of cardiovascular disease: a report of the American College of Cardiology/American heart association task force on clinical practice guidelines. Circulation. 2019;140(11):e596-e646. doi:doi:10.1161/CIR.0000000000000678

10. Mach F, Baigent C, Catapano AL, et al. 2019 ESC/EAS Guidelines for the management of dyslipidaemias: lipid modification to reduce cardiovascular risk. Eur Heart J. 2020;41(1):111-188. doi: doi:10.1093/eurheartj/ehz455

11. Grundy SM, Becker D, Clark LT, et al. Detection, evaluation, and treatment of high blood cholesterol in adults (Adult Treatment Panel III). Circulation. 2002;106(25):3143-3421. doi:doi:10.1161/circ.106. 25.3143

12. Cleeman JI. Executive summary of the third report of the National Cholesterol Education Program (NCEP) expert panel on detection, evaluation, and treatment of high blood cholesterol in adults (adult treatment panel III). J Am Med Assoc. 2001;285(19):2486-2497. doi: doi:10.1001/jama.285.19.2486

13. Assmann G, Cullen P, Schulte H. Simple scoring scheme for calculating the risk of acute coronary events based on the 10-year followup of the Prospective Cardiovascular Münster (PROCAM) study. Circulation. 2002;105(3):310-315. doi:doi:10.1161/hc0302.102575

14. Goff DC, Lloyd-Jones DM, Bennett G, et al. 2013 ACC/AHA guideline on the assessment of cardiovascular risk: A report of the American college of cardiology/American heart association task force on practice guidelines. J Am Coll Cardiol. 2014;63(25 PART B):2935-2959. doi:doi:10.1016/j.jacc.2013.11.005

15. Fowkes FG, Murray GD, Butcher I, et al. Ankle brachial index combined with framingham risk score to predict. JAMA. 2015;300 (2):197-208

16. Greenland P, LaBree L, Azen SP, Doherty TM, Detrano RC. Coronary artery calcium score combined with framingham score for risk prediction in asymptomatic individuals. $\mathrm{J} \mathrm{Am} \mathrm{Med} \mathrm{Assoc.}$ 2004;291(2):210-215. doi:doi:10.1001/jama.291.2.210

17. Polak JF, Szklo M, Kronmal RA, et al. The value of carotid artery plaque and intima-media thickness for incident cardiovascular disease: the multi-ethnic study of atherosclerosis. J Am Heart Assoc. 2013;2(2):2. doi:doi:10.1161/JAHA.113.000087

18. Betés M, Muñoz-Navas MA, Duque JM, et al. Use of colonoscopy as a primary screening test for colorectal cancer in average risk people. Am J Gastroenterol. 2003;98(12):2648-2654. doi:doi:10.1016/j. amjgastroenterol.2003.09.041
19. Lin OS, Kozarek RA, Schembre DB, et al. Risk stratification for colon neoplasia: screening strategies using colonoscopy and computerized tomographic colonography. Gastroenterology. 2006;131 (4):1011-1019. doi:doi:10.1053/j.gastro.2006.08.015

20. Yeoh KG, Ho KY, Chiu HM, et al. The Asia-Pacific Colorectal Screening score: A validated tool that stratifies risk for colorectal advanced neoplasia in asymptomatic Asian subjects. Gut. 2011;60 (9):1236-1241. doi:doi:10.1136/gut.2010.221168

21. Schroy PC, Wong JB, O'Brien MJ, Chen CA, Griffith JL. A risk prediction index for advanced colorectal neoplasia at screening colonoscopy. Am J Gastroenterol. 2015;110(7):1062-1071. doi: doi:10.1038/ajg.2015.146

22. Kim DH, Cha JM, Shin HP, Joo KR, Lee J, Park D. Development and validation of a risk stratification-based screening model for predicting colorectal advanced neoplasia in korea. J Clin Gastroenterol. 2015;49 (1):41-49. doi:doi:10.1097/MCG.0000000000000102

23. Chen G, Mao B, Pan Q, Liu Q, Xu X, Ning Y. Prediction rule for estimating advanced colorectal neoplasm risk in average-risk populations in southern Jiangsu Province. Chinese $J$ Cancer Res. 2014;26(1):4-11. doi:doi:10.3978/j.issn.1000-9604.2014. 02.03

24. Cai QC, Da YE, Xiao Y, et al. Derivation and validation of a prediction rule for estimating advanced colorectal neoplasm risk in average-risk chinese. Am J Epidemiol. 2012;175(6):584-593. doi: doi:10.1093/aje/kwr337

25. Ulmer H, Borena W, Rapp K, et al. Serum triglyceride concentrations and cancer risk in a large cohort study in Austria. $\mathrm{Br} J$ Cancer. 2009;101(7):1202-1206. doi:doi:10.1038/sj.bjc.6605264

26. Chandler PD, Song Y, Lin J, et al. Lipid biomarkers and long-term risk of cancer in the Women's Health Study. Am J Clin Nutr. 2016;103(6):1397-1407. doi:doi:10.3945/ajcn.115.124321

27. Peng L, Weigl K, Boakye D, Brenner H. Risk scores for predicting advanced colorectal neoplasia in the average-risk population: a systematic review and meta-analysis. Am $J$ Gastroenterol. 2018;113(12):1788-1800. doi:doi:10.1038/s41395-018-0209-2

28. Basyigit S, Ozkan S, Uzman M, et al. Should screening for colorectal neoplasm be recommended in patients at high risk for coronary heart disease. Med (United States). 2015;94:20. doi:doi:10.1097/MD.000 0000000000793

29. Yamaji Y, Mitsushima T, Koike K. Pulse-wave velocity, the ankle-brachial index, and the visceral fat area are highly associated with colorectal adenoma. Dig Liver Dis. 2014;46(10):943-949. doi: doi:10.1016/j.dld.2014.05.012

30. Kim J, Lee JY, Ham NS, et al. Association between carotid ultrasonography findings and colorectal adenoma in asymptomatic adults. Dig Dis Sci. 2019. doi:doi:10.1007/s10620-019-05899-7

31. Kim H, Cho YJ, Kim YA, Gwak SG. Association between colorectal adenoma and carotid atherosclerosis in Korean adults. Int J Environ Res Public Health. 2018;15(12):12. doi: doi:10.3390/ijerph15122762

32. Yun KE, Chang Y, Rampal S, et al. Coexistence of colorectal adenomas and coronary calcification in asymptomatic men and women. J Clin Gastroenterol. 2018;52(6):508-514. doi:doi:10.1097/MCG.00 00000000000824

33. Choi SH, Kang HY, Choi SY, et al. Colorectal adenoma is associated with coronary artery calcification in a Korean population. Atherosclerosis. 2015;242(2):515-520. doi:doi:10.1016/j.atherosclerosis.2015.08.004

34. Wang TJ. Assessing the role of circulating, genetic, and imaging biomarkers in cardiovascular risk prediction. Circulation. 2011;123 (5):551-565. doi:doi:10.1161/CIRCULATIONAHA.109.912568

35. Higgins JPT GS, ed. Cochrane Handbook for Systematic Reviews of Interventions Version 5.1.0 [Updated March 2011]. The Cochrane Collaboration; 2011.

36. Moher D, Liberati A, Tetzlaff J, et al. Preferred reporting items for systematic reviews and meta-analyses: the PRISMA statement. PLoS Med. 2009;6:7. doi:doi:10.1371/journal.pmed.1000097 
37. Whiting PF, Rutjes AWS, Westwood ME, et al. Quadas-2: A revised tool for the quality assessment of diagnostic accuracy studies. Ann Intern Med. 2011;155(8):529-536. doi:doi:10.7326/0003-4819-1558-201110180-00009

38. Cha JM, Lee J, Joo KR, Shin HP, Park JJ, Cho BH. May abnormal carotid intima-media thickness predict colorectal neoplasm? Hepatogastroenterology. 2011;58(109):1142-1147. doi:doi:10.5754/ hge10541

39. Yang MH, Cho J, Choi YH, Son HJ, Rhee JC, Sung J. The association between coronary artery calcification and colorectal adenoma. Hepatogastroenterology. 2013;60(123):538-542. doi:doi:10.5754/ hge12709

40. Kim HB, Lee YJ, Shim JY, Lee HR. The association between coronary calcification and adenomatous polyps of colon in Korean adults. Clin Res Hepatol Gastroenterol. 2014;38(5):649-654. doi: doi:10.1016/j.clinre.2014.01.004

41. Lee YJ, Baik SJ, Park H, et al. The association between progression of coronary artery calcium and colorectal adenoma: A retrospective follow-up study of asymptomatic Koreans. Medicine (Baltimore). 2019;98(42):e17629. doi:doi:10.1097/MD.0000000000017629

42. Lee JY, Hong SN, Kim JH, et al. Risk for coronary heart disease increases risk for colorectal neoplasm. Clin Gastroenterol Hepatol. 2013;11(6):695-702. doi:doi:10.1016/j.cgh.2012.10.017

43. Niederseer D, Bracher I, Stadlmayr A, et al. Association between cardiovascular risk and diabetes with colorectal neoplasia: a site-specific analysis. J Clin Med. 2018;7(12):484. doi:doi:10.3390/ jcm7120484

44. Ford ES, Giles WH, Mokdad AH. The distribution of 10-Year risk for coronary heart disease among U.S. adults: findings from the national health and nutrition examination survey III. J Am Coll Cardiol. 2004;43(10):1791-1796. doi:doi:10.1016/j.jacc.2003.11.061

45. Koene RJ, Prizment AE, Blaes A, Konety SH. Shared Risk Factors in Cardiovascular Disease and Cancer. Circulation. 2016;133 (11):1104-1114. doi:doi:10.1161/CIRCULATIONAHA.115.020406

46. Giovannucci E Metabolic Syndrome, Hyperinsulinemia, and Colon Cancer: A Review 1-3.; 2007. https://academic.oup.com/ajcn/articleabstract/86/3/836S/4649613. Accessed June 26, 2020.
47. Pansuria M, Xi H, Li L, Yang XF, Wang H. Insulin resistance, metabolic stress, and atherosclerosis. Front Biosci Sch. 2012;4 S (3):916-931. doi:doi:10.2741/s308

48. Handy CE, Quispe R, Pinto X, et al. Synergistic opportunities in the interplay between cancer screening and cardiovascular disease risk assessment: together we are stronger. Circulation. 2018;138 (7):727-734. doi:10.1161/CIRCULATIONAHA.118.035516

49. Wells BJ, Kattan MW, Cooper GS, Jackson L, Koroukian S. ColoRectal cancer predicted risk online (CRC-PRO) calculator using data from the multi-ethnic cohort study. J Am Board Fam Med. 2014;27(1):42-55. doi:doi:10.3122/jabfm.2014.01.130040

50. Handy CE, Quispe R, Pinto X, et al. Synergistic opportunities in the interplay between cancer screening and cardiovascular disease risk assessment: together we are stronger. Circulation. 2018;138 (7):727-734. doi:doi:10.1161/CIRCULATIONAHA.118.035516

51. Jee SH, Jang Y, Oh DJ, et al. A coronary heart disease prediction model: the Korean heart study. BMJ Open. 2014;4(5):e005025. doi: doi:10.1136/bmjopen-2014-005025

52. Liu J, Hong Y, D’Agostino RB, et al. Predictive value for the Chinese population of the Framingham CHD risk assessment tool compared with the Chinese Multi-provincial Cohort Study. J Am Med Assoc. 2004;291(21):2591-2599. doi:doi:10.1001/jama.291.21.2591

53. Leung JY, Lin SL, Lee RS, Lam TH, Schooling CM. Framingham risk score for predicting cardiovascular disease in older adults in Hong Kong. Hong Kong Med J. 2018;24 Suppl 4(4):8-11.

54. Barzi F, Patel A, Gu D, et al. Cardiovascular risk prediction tools for populations in Asia. J Epidemiol Community Health. 2007;61 (2):115-121. doi:doi:10.1136/jech.2005.044842

55. McClelland RL, Jorgensen NW, Budoff M, et al. 10-year coronary heart disease risk prediction using coronary artery calcium and traditional risk factors: derivation in the mesa (multi-ethnic study of atherosclerosis) with validation in the hnr (heinz nixdorf recall) study and the DHS (dallas heart Study). J Am Coll Cardiol. 2015;66(15):1643-1653. doi:doi:10.1016/j.jacc.2015.08.035
Clinical Epidemiology

\section{Publish your work in this journal}

Clinical Epidemiology is an international, peer-reviewed, open access, online journal focusing on disease and drug epidemiology, identification of risk factors and screening procedures to develop optimal preventative initiatives and programs. Specific topics include: diagnosis, prognosis, treatment, screening, prevention, risk factor modification,

Submit your manuscript here: https://www.dovepress.com/clinical-epidemiology-journal systematic reviews, risk \& safety of medical interventions, epidemiology \& biostatistical methods, and evaluation of guidelines, translational medicine, health policies \& economic evaluations. The manuscript management system is completely online and includes a very quick and fair peer-review system, which is all easy to use. 